\title{
LA LITTÉRATURE FRANCO-VIETNAMIENNE : \\ UNE RÉPONSE AU COLONIALISME
}

\section{Kathy V. DINH, Université McMaster}

Résumé

Dans cet article, je me propose d'examiner comment la langue française a été utilisée par les Français pour faciliter des projets impériaux, mais aussi par des écrivaines vietnamiennes comme réponse au colonialisme français. De plus, j'examinerai ce qu'implique le fait d'écrire dans une langue qui n'est pas la sienne - notamment dans la langue du colonisateur. Enfin, je discuterai l'importance de la langue, à savoir son emploi et son impact sur la culture et comment elle nous permet de comprendre la formation de l'identité du colonisé.

Avant l'arrivée des Français en Indochine dans la première moitié du XVIIe siècle, le Việt Nam avait déjà lutté pendant plus d'un millénaire pour préserver son identité nationale contre les envahisseurs étrangers. Connue autrefois sous le nom de Cochinchine en Occident, le Viêt Nam n'a cessé de se battre entre 111 av. J.-C. et 938 apr. J.-C. pour son indépendance de la Chine. Cette lutte a été suivie par un long effort en vue de protéger son unité territoriale contre les Portugais, les Hollandais, les Français, et puis les Américains. Certains croient que c'est à cause de cette longue expérience de résistance que les colonisateurs n'ont jamais réussi à démanteler ou à pénétrer complètement la culture vietnamienne. Cependant, l'expérience coloniale a toujours des implications complexes sur presque tous les aspects de la culture colonisée, ce qui mérite un examen approfondi. Par exemple, il est indéniable que la présence française coloniale au Việt Nam a laissé des traces durables sur la culture. À travers leur 
«mission civilisatrice, » les Français ont laissé un instrument qui deviendra libérateur en donnant une voix aux Vietnamiens, et qui contribuera à la restauration nationale : leur langue.

Dans l'optique de ce colloque, «les langues françaises et leur représentation », je voudrais examiner comment la langue française a été utilisée par les Français pour faciliter des projets impériaux, mais aussi par des écrivaines vietnamiennes pour ébranler l'autorité coloniale française.

Pour commencer, toute expérience coloniale suscite à la fois échange culturel et assimilation, mais aussi résistance. Bill Ashcroft, théoricien du post-colonialisme, souligne que la lecture est un aspect important du processus de résistance. Lecture ne signifie pas seulement l'action matérielle de lire, mais aussi l'interprétation herméneutique des textes et le déchiffrage de ce qui est écrit. Dans son roman Post-Colonial Transformation, Ashcroft écrit : «Resistance, as we have seen, need not necessarily mean rejection of dominant culture...the most effective post-colonial resistance has always been the wrestling, from imperial hands, of some measure of political control over such things as language, writing and various kinds of cultural discourse, the entry into the 'scene' of colonization to reveal frictions of cultural difference, to actually make use of aspects of the colonizing culture so as to generate transformative cultural production. » (Ashcroft, p. 47) Ceci nous mène à la question de la langue qui est employée par des écrivaines résistantes, notamment dans la littérature franco-vietnamienne. Afin de mieux comprendre les implications que l'usage d'une langue autre que la sienne entrâne, il nous faut d'abord examiner comment la langue d'origine a été affectée, et dans quelle mesure elle a même été remplacée par la langue colonisatrice.

Dans son livre The Vietnamese Novel in French, Jack Yeager propose que l'histoire littéraire francophone au Việt Nam a été influencée par quatre périodes majeures : la phase de 
préparation, celle du développement littéraire, celle du bouleversement politique et des conflits armés, et enfin celle de la partition du Việt Nam (Yeager, pp.46-50). La première période, qui se situe entre 1850 et 1913, a commencé avec l'introduction de la langue française au Việt Nam. Pour Yeager, l'influence exercée par les autorités coloniales a été une cause lointaine de l'émergence d'une littérature franco-vietnamienne. Il suggère que même avant la période coloniale, les personnes lettrées, notamment celles de la cour de Hué, ont utilisé le français comme langue diplomatique et littéraire. Au milieu du XIXe siècle, les autorités coloniales ont déclaré le français outil linguistique du nouveau système d'éducation. La notion de colonialisme et d'autorité coloniale, provenant de l'autorité française, s'est alors propagée à travers les écoles et les élites sociales. De cette manière, les colonisateurs ont utilisé les élites locales pour établir un contact avec la population et pour transmettre et affirmer la suprématie de la langue française. Le développement littéraire a commencé peu après 1913. La littérature moderne écrite en français a été favorisée par les partisans d'une expression innovatrice. À ce moment-là, la plupart des écrivains étaient bilingues. Le Việt Nam a ainsi été témoin d'une explosion littéraire francophone - les pièces de théâtre, la poésie, les essais traitant de la littérature et de la culture, et les mémoires de ceux qui avaient quitté le pays et leurs expériences à leur retour de France étaient tous écrits en français. Puis, des bouleversements politiques et des conflits armés ont commencé ; le Việt Nam a été subjugué par une double colonisation, celle des Français et celle des impérialistes japonais. Pendant l'occupation franco-japonaise, un nationalisme a émergé qui s'est manifesté par un appel au retour aux racines. Vers 1944, des écrivains comme Nguyen Huyen ont écrit des essais sur «la civilisation annamite» et des collections de folklore vietnamien sont apparues. La partition du Việt Nam en 1954 a créé une scission entre les démocrates de gauche (au nord) et les nationalistes modérés (au sud). Cette période a marqué la 
fin de la présence coloniale française, quoique la langue française fût encore perçue par certains comme un «instrument de progrès » et ait continué à être le deuxième moyen d'expression littéraire.

Mais pourquoi écrire en français? Les raisons sont complexes. Certains croient que la littérature franco-vietnamienne a été en partie influencée par l'intérêt que portaient les Vietnamiens à l'érudition. Il ne faut pas perdre de vue l'impact de la colonisation intellectuelle qui s'est propagée par le système d'éducation réglementé par les Français, et qui suivait les différences régionales et sociales. La bourgeoisie, d'où venaient les dirigeants, était éduquée dans un système qui dénigrait la culture vietnamienne comme étant inutile pour qui voulait vivre dans la modernité. Ainsi, des écrivains ont adopté la langue française comme moyen supérieur de communication. D'autres ont adopté la langue de l'oppresseur comme une arme, ou bien un outil pour désassembler la maison du maître de l'intérieur. La voix française devenait alors essentiellement un mécanisme d'émancipation, qu'ils utilisent pour transmettre et valider leur culture et leur civilisation aux Français et ainsi contrer toute déformation culturelle. (Yeager, p. 53) De plus, le choix de la langue est vital en ce qu'il indique pour qui la littérature a été écrite, mais aussi parce que cela crée un pont qui permet aux Vietnamiens d'atteindre leurs oppresseurs, tout en maintenant une certaine distance.

Comment exactement ont-ils utilisé cette langue comme réponse au colonialisme français? Il nous faut envisager la nature des relations de pouvoir entre le dominant et le subalterne et comment ceci se traduit dans la littérature franco-vietnamienne. Il est évident que l'oppression et la peur qu'elle génère nient aux subalternes la possibilité de retourner l'insulte. Il fallait alors créer une sphère sociale pour dire ce qu'on ne pouvait pas dire en face du pouvoir. Dans son essai Domination and the Arts of Resistance, James Scott écrit : «... slaves and serfs 
ordinarily dare not contest the terms of their subordination openly. Behind the scenes, though, they are likely to create and defend a social space in which offstage dissent to the official transcript of power relations may be voiced» (Scott, p. xi). En d'autres mots, le processus de domination génère une conduite hégémonique en public, mais aussi à l'arrière-plan un discours de résistance qui contient ce qu'il ne faut pas dire en face du pouvoir. Scott appelle ces deux discours : la transcription publique et la transcription cachée.

La transcription publique désigne les interactions ouvertes entre les subalternes et ceux qui dominent. Il est important de reconnaître qu'il est peu probable que cette transcription donne toute l'histoire des relations de pouvoir, puisque c'est souvent dans l'intérêt des deux côtés d'apparaître aimables malgré ce qu'ils ressentent. En fait, une des techniques-clés de survie pour le subalterne est l'art de la représentation ou de la performance dans des situations accablantes, à savoir celui du masque narratif. Ainsi, cette transcription cachée est utilisée comme réponse à la transcription publique. Comme les subalternes portent fréquemment des masques, c'est la transcription cachée, le discours qui se manifeste « dans les coulisses » au-delà de l'observation directe par ceux qui dominent, qui va présenter une vraie critique du pouvoir. Cependant, la transcription cachée a aussi besoin d'un public, même si le dominant ne fait pas partie de ce public. Il était important pour les résistants de créer une sphère sociale, une communauté de lecteurs séparée du contrôle et de la surveillance hégémonique pour produire leurs discours de résistance. Pour faire connaitre au public la transcription cachée qui contenait des idées subversives, les écrivains ont dû écrire leurs histoires dans un style anodin afin de garder la réalité de leur message cachée, car tout au plus les histoires avec des messages équivoques pouvaient être contées publiquement. La transcription cachée et l'usage du masque narratif se sont manifestés concrètement sous forme ironique (dire le contraire de ce qu'on veut faire 
entendre) et de discours hybrides (composés de double sens). Comme ces discours peuvent s'interpréter de plusieurs manières, c'est au lecteur d'assumer la responsabilité d'élaborer la signification appropriée. Par conséquent, le lecteur devient activement engagé dans la littérature et le processus critique lui-même. Ainsi, pour que la transmission des messages cachés se fasse avec succès, le lecteur doit être capable de reconnaître et de décoder ce discours double. Ainsi, l'usage de représentations culturelles sert un double objectif : i) il protège l'intention de l'auteur, et ii) il s'adresse directement aux lecteurs vietnamiens car, ayant souffert les mêmes injures et les mêmes humiliations, seuls eux sont en mesure de reconnaître ses représentations. Ceci marque l'apparition d'une littérature franco-vietnamienne de résistance comme réponse au colonialisme.

Comme cela a déjà été mentionné, en s'appropriant l'usage de la langue française comme moyen de communication, bien que la langue française ait été un instrument d'oppression, les écrivains vietnamiens ont trouvé une façon de l'utiliser de façon efficace pour se libérer. Dans son essai Remnants of Empire in Algeria and Vietnam, Pamela Pears souligne comment, dans le cas des colonisés, la maîtrise de la langue colonisatrice a aidé des groupes révolutionnaires à accéder aux modèles européens de féminisme et de nationalisme. Par conséquent, à cause de leur bilinguisme, ces érudites ont joué un rôle énorme dans la formation de l'identité nationale. Selon Pears, les intelligentsias (produites par des systèmes d'éducation coloniaux) ont facilité la communication entre les colonisateurs et la population indigène. De plus, plusieurs membres des forces révolutionnaires vietnamiennes se sentaient à l'aise avec l'utilisation de langue française. Même après les révolutions, il était difficile pour des nations nouvellement libérées de l'oppression française de se détacher de la langue du colonisateur, car la plupart des personnes bien éduquées étaient mieux formées en français qu'en vietnamien (Pears, p. 11). Dans son étude sur les écrivaines féministes postcoloniales, Pears dit : 
In order to express herself in French, the Francophone author, if she follows binary oppositions, is forced to divide herself between one cultural heritage and that which is intrinsic to the language in which she writes...These authors symbolize through their writing the cultural and historical splits between...Vietnam and France. In their attempts to go beyond the "broken" relationships created out of colonialism, the wars for independence, and the current political climate in which they live, we witness their fight to overcome the ideological trap of duality. (100)

Cette lutte se manifeste à travers la littérature franco-vietnamienne. La femme et son corps sont souvent utilisés de façon symbolique, représentant le pays et l'identité nationale. Plusieurs auteurs révolutionnaires qui étaient subjugués par des pouvoirs coloniaux, les sœurs Tran Nhu, Mai Van, Thu Huong Duong, Ca Nha, Thi Hoai Pham, et Thi Vang Anh Phan (auteurs vietnamiens écrivant tous en français) par exemple, se servent souvent de cette allégorie dans leurs pièces de théâtre, la poésie, les essais, et les mémoires pour illustrer leur conflit de dualité, les barrières culturelles, et l'oppression sociale et pour les dépasser.

Deux autres exemples d'auteurs post-coloniaux d'origine vietnamienne écrivant en français et qui ont utilisé la littérature franco-vietnamienne pour offrir des représentations culturelles grâce à la transcription cachée pour débattre des problèmes culturels post-coloniaux, sont Kim Lefèvre et Anna Moi. Née au Viêt-nam pendant la période de la colonisation française d'un père français et d'une mère vietnamienne, Kim Lefèvre vécut une enfance et une adolescence difficiles au Viêt-nam. Ses œuvres autobiographiques publiées à Paris, Métisse blanche (1989) et Retour à la saison des pluies (1990), racontent son histoire qui s'est déroulée dans un contexte de colonisation et d'exil. Anna Moi, née à Saigon en 1955, est retournée au 
Vietnam en 1993 après une vingtaine d'années passées en France. Écrit au Viêt-nam et publié en France, L'écho des rizières (2002) raconte sa vision intimiste du Viêt-nam actuel. Ces deux écrivaines se sont servies du masquage narratif dans un canevas post-colonial pour évoquer les lecteurs vietnamiens qui ont été maltraités, méprisés et rejetés aussi bien par les Français que par les Vietnamiens au Viêt-nam. Elles ont traité non seulement de la position des Vietnamiens pendant l'époque coloniale, mais aussi de la position des femmes de cette société, pour qui elles écrivaient. Ainsi, dans la préface de Retour à la saison des pluies, Lefèvre insère une citation de La Forteresse, de Mesa Selimovic : «Ce qu'on n'a pas écrit n'existe pas ; la mort l'a emporté. » La Forteresse est un roman qui traite de la guerre à Sarajevo, de sujets comme le traumatisme, la perte, et la mémoire sélective (ce qui vaut la peine de se rappeler, ce qui n’en vaut pas la peine) qui sert de référence à la thématique de la mémoire et de l'oubli pour la suite de Retour à la saison des pluies. L'intertextualité ici crée un lien entre le Viêt-nam et Sarajevo - deux régions reconnues pour leurs histoires de guerre et de massacre.

Dans la préface, Lefèvre écrit: «Tout changea avec la parution du livre (Métisse Blanche). En l'écrivant j'avais mis en marche, sans en avoir conscience, la machine à remonter le temps. Et les années-lumière que j'avais voulu jeter entre le Việt-Nam et moi, entre mon enfance et moi, comme un grand espace d'oubli, se retrouvèrent tout à coup abolies. » (Lefèvre, p.18) L'acte d'écrire est important car il aide à extirper des événements cruciaux du passé. Lefèvre décrit son enfance et ses souvenirs du Việt Nam comme «un grand espace d'oubli» mais il est important de discerner la différence entre ce qui est oublié et ce qui est supprimé. L'oubli est caractérisé par la disparition de souvenirs dans la mémoire tandis que la suppression ne dénote pas l'élimination, mais une inhibition de ce qui existe encore. Dans Retour à la saison des pluies, Lefèvre dévoile son objectif lorsqu'elle a écrit Métisse blanche : 
«Je l'avais fait pour exorciser le passé mais aussi pour rendre hommage à une femme vietnamienne, ma mère, une femme tout à la fois pitoyable et admirable, afin que son destin ne tombe pas dans l'oubli. Et par-dessus tout, j'avais ressenti la nécessité de parler au nom de toutes les Eurasiennes que j'avais connues, de toutes celles qui, comme moi, avaient été méprisées et rejetées aussi bien par les Français au Vietnam que par les Vietnamiens, qui vivent aujourd'hui quelque part et dont on n'a jamais entendu les voix. » (Lefèvre, p.16)

Pour elle, la littérature est un instrument non violent utilisé pour faire la critique sociale de manière anodine pour reconnaître et reconstruire le passé. Écrire de manière anodine, sur le plan littéraire, implique une écriture qui n'est pas menaçante et qui ne conduira pas à des attaques. Lefèvre voulait critiquer l'hybridité dans la société du métissage pendant les jours coloniaux de son enfance ; une hybridité qui a insisté sur la division entre le «nous » et l' «autrui »— ce qui était vietnamien et ce qui était français. Bien que nous ne soyons plus en période coloniale, le colonialisme est un processus perpétuel qui conduit à des effets psychologiques continuels. Lefèvre choisit encore d'utiliser le français pour s'adresser aux Français qui l'ont niée, et a recours aux représentations culturelles pour s'adresser aux Vietnamiens qui l'ont méprisée et rejetée. D’une façon encore plus importante, pour ces écrivaines, ceci était un moyen de valider leur souffrance pour qu'elles puissent commencer le processus de guérison. Il est important de noter ici comment le facteur «gender » affecte leur situation et leur écriture. Les femmes étaient assujetties à une double oppression : par les pouvoirs coloniaux, et par une société patriarcale. Le fait que ces deux femmes d'origine vietnamienne puissent s'exprimer en français indique qu'elles ont reçu une éducation formelle, et qu'elles venaient certainement d'une classe privilégiée. De plus, être une femme éduquée, c'est aussi appartenir à une minorité. Cependant, 
ce sont de tels groupes minoritaires qui ont donné naissance à un fort nationalisme qui s'est manifesté par un désir de retour aux racines culturelles. Ce désir, manifesté par des rappels historiques, est observable à travers les descriptions détaillées de traditions. Il est commun pour des écrivaines comme Kim Lefèvre, Anna Moi, et bien d'autres, d'évoquer des images du paysage et des styles de vie traditionnels des paysans. Ces descriptions sont importantes, car elles révèlent ce qu'est leur Việt-Nam, ce que leur pays signifie pour elles, et ce qu'il était avant d'être connu comme un pays marqué par la guerre et la colonisation, comme par leurs effets sur la culture vietnamienne.

Il est important d'étudier l'emploi de la langue et son impact sur la culture ainsi que sur la société parce que cela nous permet de comprendre la transformation de l'identité du colonisé. Dans le cas de l'histoire franco-vietnamienne, les Français ont imposé leur langue aux Vietnamiens pour les resocialiser. Puis, les Vietnamiens ont utilisé cette langue pour encourager la résistance, pour se resocialiser eux-mêmes et pour faire connaître leur désir d'un retour aux traditions qui ont défini l'identité vietnamienne. À mon avis, l'usage de la langue française a eu pour effet de renforcer l'identité nationale des Vietnamiens. Lorsqu'ils se sont rendu compte que leur culture était en train d'être remplacée par celle de l'envahisseur, et qu'ils étaient devenus des citoyens de deuxième classe dans leur propre pays, une nouvelle admiration pour leur propre héritage et un désir d'y retourner sont nés qui, sur le plan littéraire, a marqué le début de l'époque contemporaine parmi les nationalistes modérés du sud.

Kim Lefèvre et Anna Moi ne sont que deux exemples d'écrivaines qui ont utilisé la littérature franco-vietnamienne pour exhumer et reconstruire ce qui était enterré dans l'oubli collectif : toute la douleur et la souffrance endurées par un peuple qui ne voulait que la paix, la liberté et le droit à l'autodétermination. Dans L'Écho des rizières, Anna Moi écrit : «Les 
Vietnamiens ont cultivé collectivement l'oubli. Ils n’ont pas oublié pour autant. » (Moi, p.14) Pendant ces dernières décennies, on constate une diminution considérable de la littérature francovietnamienne, ce qui aura des implications inévitables sur la culture à l'avenir. Qu'est-ce que cela veut dire, de perdre une forme de littérature, et essentiellement, une partie de la culture qui a joué un rôle intrinsèque dans l'émancipation du Việt-Nam vers son indépendance ? À mon avis, c'est une impasse. Il est vrai que dans une certaine mesure le Việt-Nam sera toujours affecté par le colonialisme. La langue française était un instrument d'oppression, mais les Vietnamiens ont trouvé une façon de l'utiliser de façon efficace pour se libérer. Le paradoxe est que le but d'écrire et de reconstruire est de pouvoir chasser les démons et guérir. Bien qu'il soit souvent difficile de revisiter certains moments du passé, les écrivaines vietnamiennes écrivent pour arrêter la douleur, mais lorsqu'elles arrêtent d'écrire, notamment en français, elles perdent une partie d'elles-mêmes. Aujourd'hui, les Vietnamiens qui choisissent d'écrire en français sont rares. De plus, en faisant des recherches pour ce travail, j'ai trouvé que les textes qui étaient écrits pendant le mouvement réactionnaire sont très difficiles à obtenir. C'est dommage parce que c'est parmi ces textes et le discours qui se fait «dans les coulisses » - la transcription cachée - que les écrivains ont présenté une critique fidèle du pouvoir et du colonialisme. Comme Mesa Selimovic l'a écrit : «Ce qu'on n'a pas écrit n'existe pas ; la mort l'a emporté. » Il y a un danger dans l'oubli. Il faut donc continuer à écrire.

\section{Bibliographie}

Ashcroft, Bill. Post-colonial Transformation. Londres, New York : Routledge, 2001.

Lefèvre, Kim. Métisse blanche. La Tour d'Aigues : Éditions de l'Aube, 2003.

---. Retour à la saison des pluies. Paris : Bernard Barrault, 1990.

Moi, Anna. L'Écho des rizières. La Tour d'Aigues : Éditions de l'Aube, 2002. 
Pears, Pamela. Remnants of Empire in Algeria and Vietnam : Women, Words, and war. Lanham : Lexington, 2004.

Scott, James. Domination and the Arts of Resistance. New Haven : Yale UP, 1990.

Yeager, Jack. The Vietnamese Novel in French : A Literary Response to Colonialism. Hanover : UP of New England, 1987. 